\title{
GW170817: Constraining the nuclear matter equation of state from the neutron star tidal deformability
}

\author{
Tuhin Malik, ${ }^{1, *}$ N. Alam, ${ }^{2,3}$ M. Fortin, ${ }^{4}$ C. Providência, ${ }^{5}$ B. K. Agrawal, ${ }^{2,6}$ T. K. Jha, ${ }^{1}$ Bharat Kumar, ${ }^{7,6}$ and S. K. Patra ${ }^{7,6}$ \\ ${ }^{1}$ BITS-Pilani, Dept. of Physics, K.K. Birla Goa Campus, GOA - 403726, India \\ ${ }^{2}$ Saha Institute of Nuclear physics, Kolkata 700064, India \\ ${ }^{3}$ Theoretical Physics Division, Variable Energy Cyclotron Centre, 1/AF Bidhannagar, Kolkata 700064, India \\ ${ }^{4}$ N. Copernicus Astronomical Center, Polish Academy of Science, Bartycka, 18, 00-716 Warszawa, Poland \\ ${ }^{5}$ CFisUC, Department of Physics, University of Coimbra, 3004-516 Coimbra, Portugal \\ ${ }^{6}$ Homi Bhabha National Institute, Anushakti Nagar, Mumbai - 400094, India \\ ${ }^{7}$ Institute of Physics, Bhubaneswar - 751005, India
}

(Received 28 February 2018; published 28 September 2018)

\begin{abstract}
Constraints set on key parameters of the nuclear matter equation of state (EoS) by the values of the tidal deformability, inferred from GW170817, are examined by using a diverse set of relativistic and nonrelativistic mean-field models. These models are consistent with bulk properties of finite nuclei as well as with the observed lower bound on the maximum mass of neutron star $\approx 2 M_{\odot}$. The tidal deformability shows a strong correlation with specific linear combinations of the isoscalar and isovector nuclear matter parameters associated with the EoS. Such correlations suggest that a precise value of the tidal deformability can put tight bounds on several EoS parameters, in particular on the slope of the incompressibility and the curvature of the symmetry energy. The tidal deformability obtained from the GW170817 and its UV, optical and infrared counterpart sets the radius of a canonical $1.4 M_{\odot}$ neutron star to be $11.82 \leqslant R_{1.4} \leqslant 13.72 \mathrm{~km}$.
\end{abstract}

DOI: 10.1103/PhysRevC.98.035804

\section{INTRODUCTION}

The physics of dense matter relevant to neutron stars (NSs) is poorly understood at present [1]. Neutron stars are made of incredibly dense matter reaching densities up to few times the nuclear saturation density $\left(\rho_{0} \approx 0.16 \mathrm{fm}^{-3}\right)$ in the core region. The NS structure depends predominantly on the nuclear equation of state $(\mathrm{EoS})$. Due to the lack of detailed knowledge of the nuclear interactions at densities typical of the NS interior, many theoretical models of the nuclear EoS have been proposed. Matter at supranuclear densities, as encountered in the NS interior, is difficult to access in terrestrial experiments. Inputs from astrophysical observations are, therefore, crucial in constraining the dense matter EoS. Currently, the most stringent constraint comes from the observation of NSs with $\approx 2 M_{\odot}[2,3]$, which sets a lower limit for the maximum mass to be predicted by an EoS.

Because NSs are massive and compact astrophysical objects, the coalescence of binary NS systems is one of the most promising sources of gravitational waves (GWs) observable by ground-based detectors [4-9]. The GW signals emitted during a NS merger depends on the behavior of neutron star matter at high densities $[10,11]$. Therefore, its detection opens the possibility of constraining the nuclear matter parameters characterizing the EoS. A significant signature carried by GWs is the tidal deformability (polarizability) of the NS, and it is well explored analytically [12-16]. In a coalescing binary NS system, during the last stage of inspiral, each NS

\footnotetext{
*tuhin.malik@gmail.com
}

develops a mass quadrupole due to the extremely strong tidal gravitational field induced by the other NS forming the binary. The dimensionless tidal deformability $\Lambda$ describes the degree of deformation of a NS due to the tidal field of the companion NS and is sensitive to the nature of the EoS.

In August, 2017, the Advanced LIGO and Advanced Virgo gravitational-wave observatories detected for the first time GWs emitted from a binary NS inspiral [17]. Remarkably, this discovery opened a new window in the field of multimessenger astronomy and nuclear physics, which revealed the potential to directly probe the physics of NSs and of the synthesis of heavy elements in the rapid neutron-capture process ( $r$ process) $[18,19]$. The analysis of GW170817 data has allowed us to put an upper bound on the NSs combined dimensionless tidal deformability with $90 \%$ confidence by using spin magnitudes consistent with the observed neutron star population. In the analysis, results for both a high-spin and a low-spin prior have been obtained to the same level of confidence. In our study we consider the constraints set by the low-spin prior because they are consistent with the masses of all known binary neutron star systems. This prior predicts that the combined dimensionless tidal deformability of the NS merger is $\tilde{\Lambda} \leqslant 800$. In Ref. [20], a reanalysis of the gravitational-wave observations of the binary neutron star merger GW170817 was done by assuming the same EoS for both stars and supplementing the gravitation-wave observation with information on the source location and distance from electromagnetic observations. For the low-spin prior these authors obtained the constraint $\tilde{\Lambda} \leqslant 1000$. On the other end, the investigation of the UV-optical-infrared counterpart of GW170817 with kilonova models and complemented with 
numerical relativity results in Ref. [21] has set a lower bound on $\tilde{\Lambda}$, i.e., $\tilde{\Lambda}>400$. It should, however, be mentioned that this lower bound was obtained from 29 merger simulations covering several masses such that $q \gtrsim 0.85$ [22] and restricted to three models of nuclear matter, one including also the $\Lambda$ hyperon. We show that these bounds on $\tilde{\Lambda}$ can be employed to deduce the respective bounds on the tidal deformability of a NS with mass $1.4 M_{\odot}$.

Studies of the correlations between nuclear matter parameters and the tidal deformability, based on a few selected relativistic mean-field models, have shown that measurements of the latter can constrain the high-density behavior of the nuclear symmetry energy [23] as well as put bounds on the value of neutron skin thickness [24]. These preliminary studies need to be validated further by using a more diverse set of models for the nuclear EoS. In earlier studies it was found that correlations between the various properties of NS and nuclear matter EoS parameters are significantly affected when a more diverse set of models are employed [25,26]. Recently, astrophysical observations of NSs, in particular, the maximum mass, the radius of a canonical $1.4 M_{\odot} \mathrm{NS}$, and the tidal deformability, have been used to constrain various parameters of the EoS [27]. However, within their assumptions, they found that the tidal deformability obtained from GW170817 is not very restrictive.

The present communication is an attempt, in view of the recent observation GW170817, to further explore the dependence of the tidal deformability on the various nuclear matter parameters describing the EoS. We study the correlations of the tidal deformability parameter with the several different nuclear matter parameters associated with a EoS by employing a representative set of relativistic mean-field (RMF) models and of Skyrme Hartree-Fock (SHF) models. The considered EoS parameters are the nuclear matter incompressibility coefficient, the symmetry energy coefficient, and their derivatives at the saturation density. We also study the model dependence of the Love number $k_{2}$, which plays a crucial role in determining the value of tidal deformability.

This paper is organized as follows: In Sec. II, we briefly outline the procedure for computing the tidal deformability and also define the various nuclear matter parameters which can be calculated for a given EoS. In Sec. III we present the EoSs for our representative set of RMF and SHF models and use them to calculate the tidal deformability and the Love number over a wide range of NS masses. The main results for the correlations of the tidal deformability, Love number, and NS radius with different nuclear matter parameters are discussed in Sec. IV. Finally, the conclusions are drawn in Sec. V.

Conventions. We have taken the value of $G=c=1$ throughout the manuscript.

\section{FRAMEWORK}

In this section, we outline the expressions required to compute the tidal deformability for a given EoS. We also define the various nuclear matter parameters that characterize the EoS.

\section{A. Tidal deformability}

The tidal deformability parameter $\lambda$ is defined as $[12,13,16,28]$

$$
Q_{i j}=-\lambda \mathcal{E}_{i j},
$$

where $Q_{i j}$ is the induced quadrupole moment of a star in a binary due to the static external tidal field $\mathcal{E}_{i j}$ of the companion star. The parameter $\lambda$ can be expressed in terms of the dimensionless quadrupole tidal Love number $k_{2}$ as

$$
\lambda=\frac{2}{3} k_{2} R^{5},
$$

where $R$ is the radius of the NS. The value of $k_{2}$ is typically in the range $\simeq 0.05-0.15[13,16,29]$ for NSs and depends on the stellar structure. This quantity can be calculated by using the following expression [13]:

$$
\begin{aligned}
k_{2}= & \frac{8 C^{5}}{5}(1-2 C)^{2}\left[2+2 C\left(y_{R}-1\right)-y_{R}\right]\left\{2 C \left[6-3 y_{R}\right.\right. \\
& \left.+3 C\left(5 y_{R}-8\right)\right]+4 C^{3}\left[13-11 y_{R}+C\left(3 y_{R}-2\right)\right. \\
& \left.+2 C^{2}\left(1+y_{R}\right)\right]+3(1-2 C)^{2}\left[2-y_{R}+2 C\left(y_{R}-1\right)\right] \\
& \times \ln (1-2 C)\}^{-1},
\end{aligned}
$$

where $C(\equiv m / R)$ is the compactness parameter of the star of mass $m$. The quantity $y_{R}[\equiv y(R)]$ can be obtained by solving the following differential equation:

$$
r \frac{d y(r)}{d r}+y(r)^{2}+y(r) F(r)+r^{2} Q(r)=0,
$$

with

$$
\begin{aligned}
F(r)= & \frac{r-4 \pi r^{3}[\epsilon(r)-p(r)]}{r-2 m(r)}, \\
Q(r)= & \frac{4 \pi r\left[5 \epsilon(r)+9 p(r)+\frac{\epsilon(r)+p(r)}{\partial p(r) / \partial \epsilon(r)}-\frac{6}{4 \pi r^{2}}\right]}{r-2 m(r)} \\
& -4\left[\frac{m(r)+4 \pi r^{3} p(r)}{r^{2}[1-2 m(r) / r]}\right]^{2} .
\end{aligned}
$$

In the previous equations, $m(r)$ is the mass enclosed within the radius $r$, and $\epsilon(r)$ and $p(r)$ are, respectively, the energy density and pressure in terms of the radial coordinate $r$ of a star. These quantities are calculated within the nuclear matter model chosen to describe the stellar EoS. For a given EoS, Eq. (4) can be integrated together with the TolmanOppenheimer-Volkoff equations [30] with the boundary conditions $y(0)=2, p(0)=p_{c}$, and $m(0)=0$, where $y(0), p_{c}$, and $m(0)$ are the dimensionless quantity, pressure, and mass at the center of the NS, respectively. One can then define the dimensionless tidal deformability: $\Lambda=\frac{2}{3} k_{2} C^{-5}$. The tidal deformabilities of the NSs present in the binary neutron star system can be combined to yield the weighted average as

$$
\tilde{\Lambda}=\frac{16}{13} \frac{(12 q+1) \Lambda_{1}+(12+q) q^{4} \Lambda_{2}}{(1+q)^{5}},
$$

where $\Lambda_{1}$ and $\Lambda_{2}$ are the individual tidal deformabilities corresponding to the two components in the NS binary with masses $m_{1}$ and $m_{2}$, respectively [12,31] with $q=m_{2} / m_{1}<1$. 


\section{B. Nuclear matter parameters}

The energy per nucleon at a given density $\rho=\rho_{n}+\rho_{p}$ with $\rho_{n}$ and $\rho_{p}$ being the neutron and proton densities, respectively, and asymmetry $\delta=\left(\rho_{n}-\rho_{p}\right) / \rho$, can be decomposed, to a good approximation, into the EoS for symmetric nuclear matter $e(\rho, 0)$, and the density-dependent symmetry energy coefficient $S(\rho)$ :

$$
e(\rho, \delta) \simeq e(\rho, 0)+S(\rho) \delta^{2} .
$$

Expanding the isoscalar contribution to third order and the isovector to second order, we obtain for the isoscalar part $e(\rho, 0)$ :

$$
e(\rho, 0)=e\left(\rho_{0}\right)+\frac{K_{0}}{2} x^{2}+\frac{Q_{0}}{6} x^{3}+O\left(x^{4}\right),
$$

and for the isovector part $S(\rho)$ :

$$
S(\rho)=J_{0}+L_{0} x+\frac{K_{\mathrm{sym}, 0}}{2} x^{2}+O\left(x^{3}\right),
$$

where $x=\frac{\rho-\rho_{0}}{3 \rho_{0}}$ and $J_{0}=S\left(\rho_{0}\right)$ is the symmetry energy at the saturation density. The incompressibility $K_{0}$, the skewness coefficient $Q_{0}$, the symmetry energy slope $L_{0}$, and its curvature $K_{\mathrm{sym}, 0}$ evaluated at saturation density are defined in, e.g., Ref. [32]. The slope of the incompressibility, $M_{0}$, at saturation density is defined as [25]

$$
M_{0}=12 K_{0}+Q_{0} .
$$

In Sec. IV we consider the correlations of the tidal deformability of NSs with the various nuclear matter parameters of the EoS: $K_{0}, Q_{0}, M_{0}, J_{0}, L_{0}, K_{\text {sym }, 0}$.

\section{EQUATION OF STATE AND TIDAL DEFORMABILITY}

In the present section we introduce a set of relativistic and nonrelativistic nuclear models that are constrained by the bulk properties of finite nuclei and the observed lower bound on the NS maximum mass. For these models we show how the tidal deformability and Love number behave over a wide range of NS masses.

\section{A. Nuclear matter equation of state}

The correlations of the properties of neutron stars with the various nuclear matter parameters of the EoS are studied by using a set of eighteen relativistic and twenty-four nonrelativistic nuclear models. These models have been employed for the study of finite nuclei and NS properties. Our set of models are based on RMF and SHF frameworks. The RMF models employed are BSR2, BSR3, BSR6 [33,34], FSU2 [35], GM1 [36], NL3 [37], NL3 $\sigma \rho 4$, NL3 $\sigma \rho 6$ [38], NL3 $\omega \rho 02$ [39], NL3 $\omega \rho 03$ [40], TM1 [41], TM1-2 [42] DD2 [43], DDH $\delta$ [44], DDH $\delta$ Mod [45], DDME1 [46], DDME2 [47], and TW [48]. The SHF models considered are the SKa, SKb [49], SkI2, SkI3, SkI4, SkI5 [50], SkI6 [51], Sly2, Sly9 [52], Sly230a [53], Sly4 [54], SkMP [55], SKOp [56], KDE0V1 [57], SK255, SK272 [58], Rs [59], BSk20, BSk21 [60], BSk22, BSk23, BSk24, BSk25, and BSk26 [61]. The values of the nuclear matter properties, such as $K_{0}, Q_{0}, M_{0}, J_{0}, L_{0}$, and $K_{\text {sym }, 0}$ vary over a wide range for our representative set
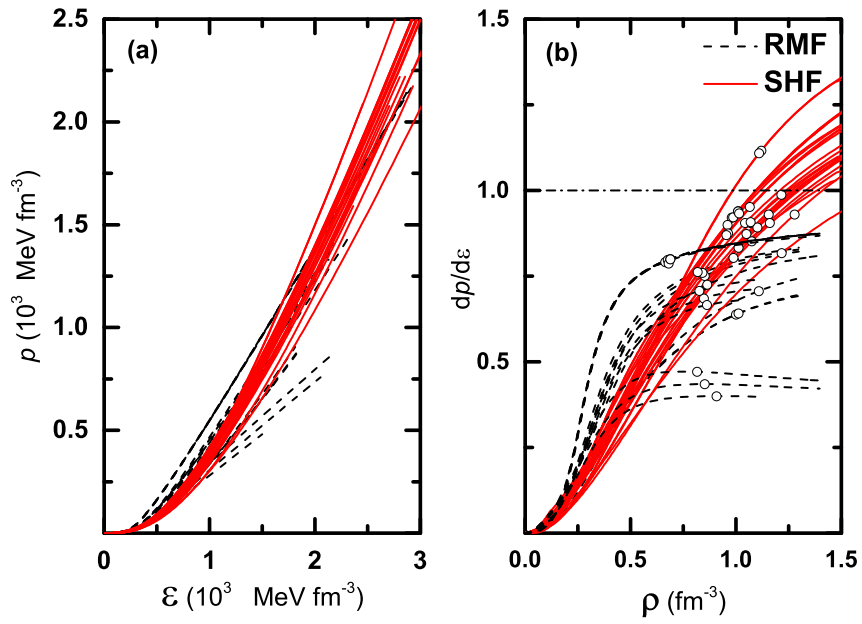

FIG. 1. Plots for (a) pressure $p$ as a function of energy density and (b) $d p / d \varepsilon$ as a function of the baryonic number density for betaequilibrated NS matter obtained by using a representative set of RMF (black dashed lines) and SHF models (red lines). The circles in the right panel correspond to the central densities and the slopes $d p / d \varepsilon$ at the maximum NS mass for each of the EoS. The BSk20 and BSk26 EoSs are marginally acausal at the NS maximum masses $\approx 2.2 M_{\odot}$ $[26,62]$.

of EoSs as can be seen from the supplementary material of Ref. [62]. As the mass of the stars in the GW170817 binary is $1.6 M_{\odot}$ or smaller, we only consider nucleonic degrees of freedom. However, a NS with a mass of $1.6 M_{\odot}$ could have non-nucleonic degrees of freedom [33,63].

The EoSs considered for all the models are consistent with the observational constraint provided by the existence of $2 M_{\odot}$ NSs [26,62]. Moreover, the considered SHF models do not become acausal for masses below $2 M_{\odot}$. We have taken a unified inner-crust core EoS for all the models [26] and the EoS of Baym-Pethick-Sutherland [64] is used for the outer crust.

In Fig. 1, we plot for NS matter the variation of pressure $p$ with the energy density $\varepsilon$ in the left panel and the variation of $d p / d \varepsilon$ with the baryon number density in the right panel for our representative set of models. The black circles denote the central density corresponding to the NS maximum mass for each EoS. The dashed line indicates the causality limit (i.e., $d p / d \varepsilon=1)$. The values of $d p / d \varepsilon$ for SHF models are larger at higher densities $\left(\rho \gg \rho_{0}\right)$ than those for the RMF models. The maximum mass NS configurations of all models studied are within the causality limit except for BSk20 and BSk26 EoSs, which are marginally acausal.

\section{B. Dependence of tidal deformability on equation of state}

One of the main focuses of the present work is to study the sensitivity of the tidal deformability to the properties of nuclear matter at saturation density. To facilitate our discussions in the next section, in Fig. 2 the dimensionless tidal deformability $\Lambda$ (left) and tidal Love number $k_{2}$ (right) obtained for our set of EoSs are plotted as a function of the NS mass. The values of $k_{2}$ show a noticeable spread across the various models. For instance, at $1.4 M_{\odot}$, the values of $k_{2}$ are in 

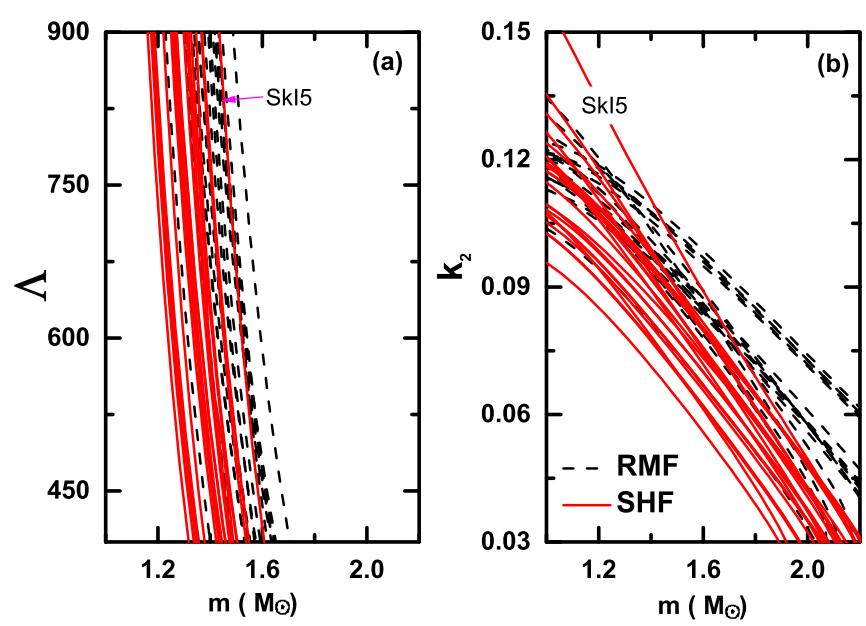

FIG. 2. (a) Tidal deformability $\Lambda$ and (b) Love number $k_{2}$ as a function of NS mass $(m)$ for a representative set of relativistic and nonrelativistic models. The SHF model, SkI5, displays markedly different behavior for $\Lambda$ as well as for $k_{2}$.

the range of 0.07 to 0.11 . For the smaller masses the spread in $k_{2}$ is larger for the SHF models, but for the larger masses RMF models give, on average, larger values of $k_{2}$. One can also see from Fig. 1 of Ref. [62] that the RMF models predict larger radii; in particular for large NS masses. Consequently, the parameter $\Lambda$ tends to be larger for the RMF models than for the SHF models. In the following, we examine the dependence of $\Lambda$ on both $k_{2}$ and $R$ in detail.

In Fig. 3 we plot the tidal deformabilities in the phase space of $\Lambda_{1}$ and $\Lambda_{2}$ associated, respectively, with the high-mass $m_{1}$ and low-mass $m_{2}$ components of the binary, for all the considered RMF and SHF models. The curves corresponding to every EoS are obtained by varying the high mass $\left(m_{1}\right)$ independently in the range $1.365<m / \mathrm{M}_{\odot}<1.60$ obtained

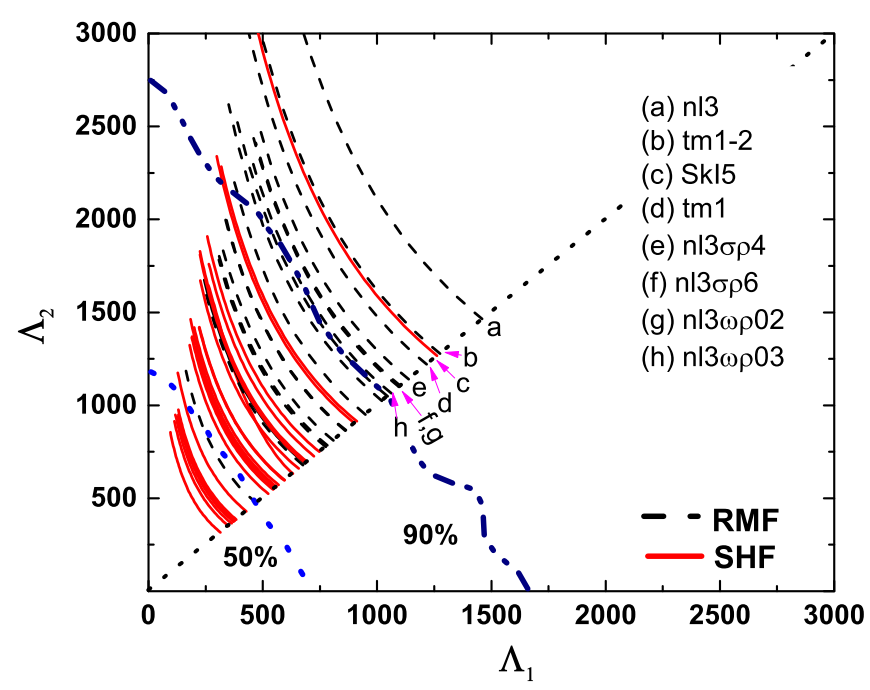

FIG. 3. Tidal deformability parameters for the case of high-mass $\left(\Lambda_{1}\right)$ and low-mass $\left(\Lambda_{2}\right)$ components of the observed GW170817. The $90 \%$ (dot-dot-dashed) and 50\% (dotted) confidence lines are taken from Ref. [17] and correspond to the low-spin priors. for GW170817 whereas the low mass $\left(m_{2}\right)$ is determined by keeping the chirp mass $\mathcal{M}=\left(m_{1} m_{2}\right)^{3 / 5}\left(m_{1}+m_{2}\right)^{-1 / 5}$ fixed at the observed value $1.188 M_{\odot}$ [17]. The dot-dot-dashed and the dotted lines represent, respectively, the $90 \%$ and $50 \%$ confidence limits obtained from the GW170817 for the low-spin priors. One can note that the $90 \%$ confidence limit suggests that SkI5 and the family of models NL3X and TM1X are ruled out except for NL3 $\omega \rho 03$. For SkI5, the values of $M_{0}$ and $L_{0}$ are 2745 and $129 \mathrm{MeV}$, respectively. For the NL3X family, the value of $M_{0}$ is larger than $3400 \mathrm{MeV}$ and $L_{0}$ is in the range of 55-70 MeV except for the base model NL3. Whereas, for TM1X family, the value of $M_{0} \approx 3100 \mathrm{MeV}$ and $L_{0} \approx 110 \mathrm{MeV}$. This indicates that a very high value of $M_{0}$ and/or $L_{0}$ may not be favored by GW170817.

\section{RESULTS AND DISCUSSIONS}

In the present section, we study the correlations of the tidal deformability $\Lambda$, the Love number $k_{2}$, and the radius of NSs $R$ with various nuclear matter parameters. As already mentioned in Sec. I, we consider the constraints from the properties of the binary neutron star that satisfy the low-spin prior [17]. In our analysis, the correlation between a pair of quantities is quantified in terms of Pearson's correlation coefficient, denoted $\mathcal{R}$ [65]. The magnitude of $\mathcal{R}$ is at most unity, indicating that the pair of quantities is completely correlated to each other. For $|\mathcal{R}|<0.5$, the correlations are usually said to be weak.

We calculate the values of the coefficients for the correlation of $\Lambda, k_{2}$, and $R$ with the nuclear matter saturation parameters $K_{0}, Q_{0}, M_{0}, J_{0}, L_{0}, K_{\mathrm{sym}, 0}$ and with several linear combinations of two parameters; in particular with $K_{0}+\alpha L_{0}$, $M_{0}+\beta L_{0}$, and $M_{0}+\eta K_{\text {sym }, 0}$. The values of $\alpha, \beta$, and $\eta$ are obtained so that, for each NS mass, they yield optimum correlations. Our correlation systematics is determined for NS masses in the range of $1.2 M_{\odot}-1.6 M_{\odot}$ since, for analysis of the low-spin prior, these masses are close to those involved in the GW170817 event. The results for the values of the $\mathcal{R}$ obtained for the correlation of $\Lambda, k_{2}$, and $R$ with individual nuclear matter parameters are presented in Table I. Table II contains the results obtained by using the linear combinations of the nuclear matter parameters. Figure 4 is the pictorial representation of the results presented in Tables I and II. Only the cases with the correlation coefficients $\mathcal{R}>0.5$ are displayed. We see from Table I that, for most of the cases, individual EoS parameters seem to be weakly or moderately correlated with the $\Lambda, k_{2}$, and $R$. Exceptionally, the $\Lambda$ and $R$ are strongly correlated with the individual nuclear matter parameters $L_{0}$ and $M_{0}$ for the NS masses $1.2 M_{\odot}$ and $1.6 M_{\odot}$, respectively. Let us point out that the correlation between the radius of low-mass NSs and the neutron skin of ${ }^{208} \mathrm{~Pb}$, which is itself correlated with $L_{0}$, was first discussed in Refs. $[39,66]$. It is seen from Table II that $\Lambda$ and $R$ are strongly correlated with $M_{0}+\beta L_{0}$ and $M_{0}+\eta K_{\mathrm{sym}, 0}$ over the wide range of NS masses considered: $\mathcal{R}$ is of the order of 0.9 . The Love number $k_{2}$ is strongly correlated with $M_{0}+\eta K_{\text {sym }, 0}$. The values of $\alpha, \beta$, and $\eta$ decrease monotonically with the NS mass. This indicates that the density dependence of symmetry energy is less important in determining the values of $\Lambda$ and $R$ at higher NS masses. The mass dependence of $\alpha, \beta$, and $\eta$ is 
TABLE I. The Pearson correlation coefficients $\mathcal{R}$ obtained for the correlations between various NS and nuclear matter properties. The values of tidal deformability $\Lambda$, radius $R$ and the Love number $k_{2}$ are evaluated for the NS masses $1.2 M_{\odot}-1.6 M_{\odot}$. The nuclear matter incompressibility $K_{0}$, skewness $Q_{0}$, slope of incompressibility $M_{0}$, symmetry energy $J_{0}$, slope of symmetry energy $L_{0}$, and curvature parameters $K_{\mathrm{sym}, 0}$ at saturation density.

\begin{tabular}{ccccccc}
\hline \hline & $K_{0}$ & $Q_{0}$ & $M_{0}$ & $J_{0}$ & $L_{0}$ & $K_{\text {sym }, 0}$ \\
\hline$\Lambda_{1.2}$ & 0.68 & 0.46 & 0.68 & 0.58 & 0.81 & 0.76 \\
$\Lambda_{1.3}$ & 0.69 & 0.51 & 0.72 & 0.56 & 0.76 & 0.74 \\
$\Lambda_{1.4}$ & 0.70 & 0.57 & 0.76 & 0.53 & 0.71 & 0.71 \\
$\Lambda_{1.5}$ & 0.71 & 0.62 & 0.80 & 0.50 & 0.65 & 0.68 \\
$\Lambda_{1.6}$ & 0.71 & 0.66 & 0.82 & 0.46 & 0.59 & 0.64 \\
$R_{1.2}$ & 0.65 & 0.48 & 0.67 & 0.65 & 0.82 & 0.70 \\
$R_{1.3}$ & 0.66 & 0.51 & 0.70 & 0.62 & 0.79 & 0.70 \\
$R_{1.4}$ & 0.67 & 0.54 & 0.72 & 0.59 & 0.75 & 0.69 \\
$R_{1.5}$ & 0.68 & 0.57 & 0.75 & 0.56 & 0.72 & 0.68 \\
$R_{1.6}$ & 0.68 & 0.60 & 0.77 & 0.53 & 0.68 & 0.66 \\
$k_{2,1.2}$ & 0.57 & 0.34 & 0.54 & -0.03 & 0.44 & 0.79 \\
$k_{2,1.3}$ & 0.62 & 0.47 & 0.65 & 0.02 & 0.43 & 0.76 \\
$k_{2,1.4}$ & 0.64 & 0.55 & 0.72 & 0.05 & 0.39 & 0.72 \\
$k_{2,1.5}$ & 0.65 & 0.63 & 0.77 & 0.08 & 0.36 & 0.66 \\
$k_{2,1.6}$ & 0.58 & 0.59 & 0.71 & 0.06 & 0.26 & 0.57 \\
\hline \hline
\end{tabular}

discussed in some detail in the appendix where, in particular, an exponential dependence of these parameters on the NS mass is proposed. As an example, in Fig. 5 we plot $M_{0}+\beta L_{0}$
TABLE II. Values of coefficients $\mathcal{R}$ obtained for the correlations of $\Lambda, R$, and $k_{2}$ with various linear combinations of EoS parameters. The calculations are performed for the NS masses $1.2 M_{\odot}-1.6 M_{\odot}$.

\begin{tabular}{|c|c|c|c|c|c|c|}
\hline & \multicolumn{2}{|c|}{$K_{0}+\alpha L_{0}$} & \multicolumn{2}{|c|}{$M_{0}+\beta L_{0}$} & \multicolumn{2}{|c|}{$M_{0}+\eta K_{\mathrm{sym}, 0}$} \\
\hline & $\mathcal{R}$ & $\alpha$ & $\mathcal{R}$ & $\beta$ & $\mathcal{R}$ & $\eta$ \\
\hline$\Lambda_{1.2}$ & 0.88 & 1.16 & 0.94 & 21.22 & 0.92 & 6.34 \\
\hline$\Lambda_{1.3}$ & 0.86 & 0.93 & 0.93 & 17.05 & 0.94 & 5.55 \\
\hline$\Lambda_{1.4}$ & 0.83 & 0.74 & 0.92 & 13.68 & 0.95 & 4.83 \\
\hline$\Lambda_{1.5}$ & 0.80 & 0.59 & 0.92 & 10.91 & 0.95 & 4.18 \\
\hline$\Lambda_{1.6}$ & 0.77 & 0.45 & 0.91 & 8.54 & 0.95 & 3.62 \\
\hline$R_{1.2}$ & 0.88 & 1.33 & 0.94 & 21.75 & 0.88 & 5.64 \\
\hline$R_{1.3}$ & 0.86 & 1.14 & 0.93 & 19.07 & 0.90 & 5.33 \\
\hline$R_{1.4}$ & 0.84 & 0.98 & 0.93 & 16.62 & 0.91 & 5.00 \\
\hline$R_{1.5}$ & 0.82 & 0.84 & 0.92 & 14.38 & 0.92 & 4.65 \\
\hline$R_{1.6}$ & 0.80 & 0.71 & 0.91 & 12.32 & 0.93 & 4.31 \\
\hline$k_{2,1.2}$ & 0.62 & 0.40 & 0.64 & 11.18 & 0.88 & 9.15 \\
\hline$k_{2,1.3}$ & 0.64 & 0.25 & 0.70 & 7.22 & 0.91 & 6.83 \\
\hline$k_{2,1.4}$ & 0.65 & 0.16 & 0.75 & 4.81 & 0.92 & 5.31 \\
\hline$k_{2,1.5}$ & 0.66 & 0.10 & 0.79 & 3.34 & 0.93 & 4.20 \\
\hline$k_{2,1.6}$ & 0.65 & 0.04 & 0.81 & 2.14 & 0.93 & 3.52 \\
\hline
\end{tabular}

and $M_{0}+\eta K_{\text {sym, } 0}$ as a function of $k_{2}$ and $\Lambda$ for a $1.4 M_{\odot}$ NS. Since $\Lambda_{1.4}$ is not very well correlated individually with $M_{0}, L_{0}$, and $K_{\text {sym }, 0}$, its strong correlation with $M_{0}+\beta L_{0}$ and $M_{0}+\eta K_{\mathrm{sym}, 0}$ is of particular importance. The values of the correlation coefficients given in the figure are obtained with the entire set of RMF and SHF models as presented in
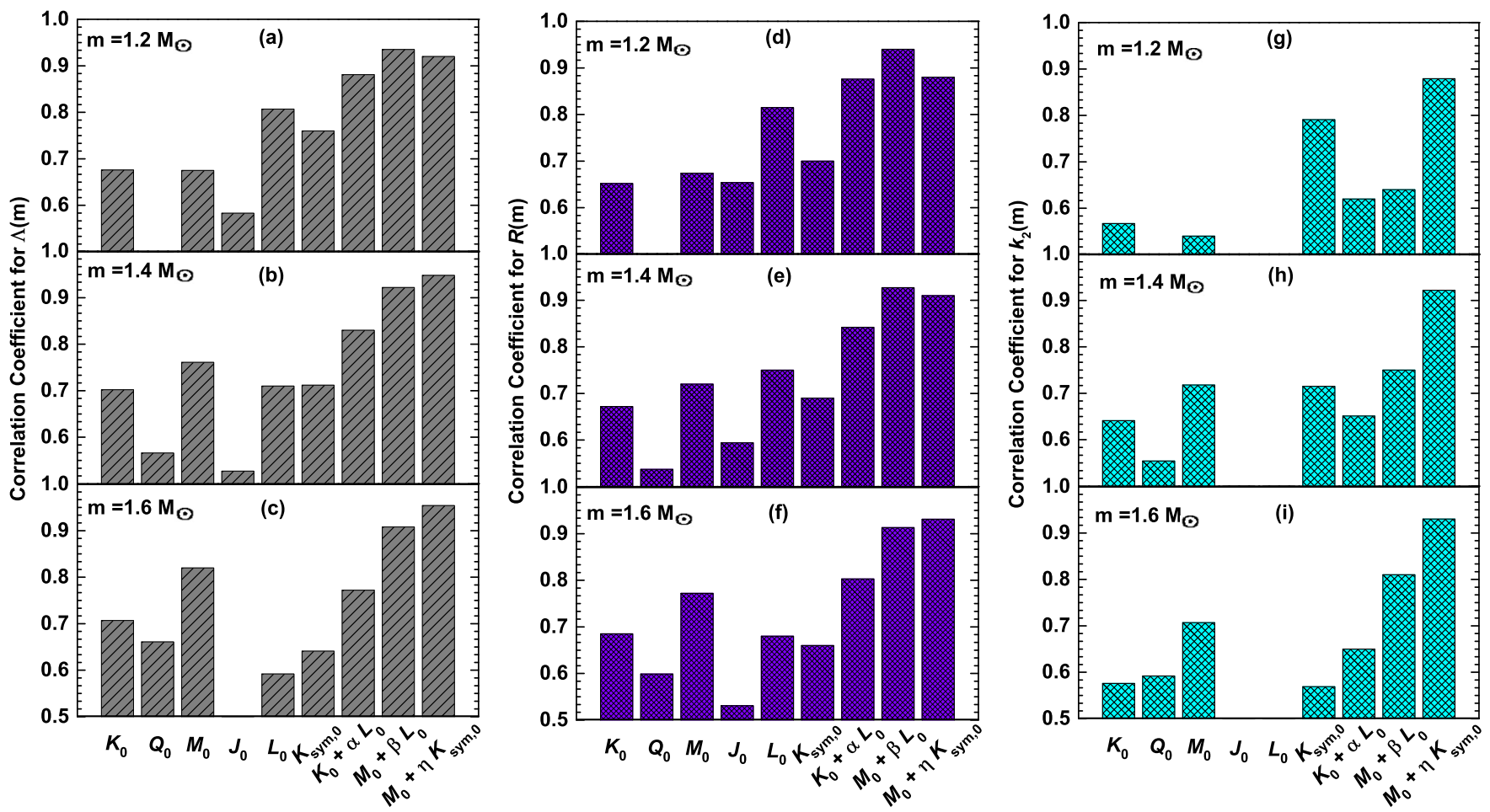

FIG. 4. Correlation coefficients $\mathcal{R}$ for (a)-(c) the tidal deformability $\Lambda$, (d)-(f) the radius $R$, and (g)-(i) the Love number $k_{2}$ with different individual nuclear matter parameters as well as with some selected linear combinations of them obtained for the NS masses $1.2 M_{\odot}$ (top), $1.4 M_{\odot}$ (middle), and $1.6 M_{\odot}$ (bottom). Results are plotted only for the cases with $\mathcal{R}>0.5$ (see Tables I and II for details). 

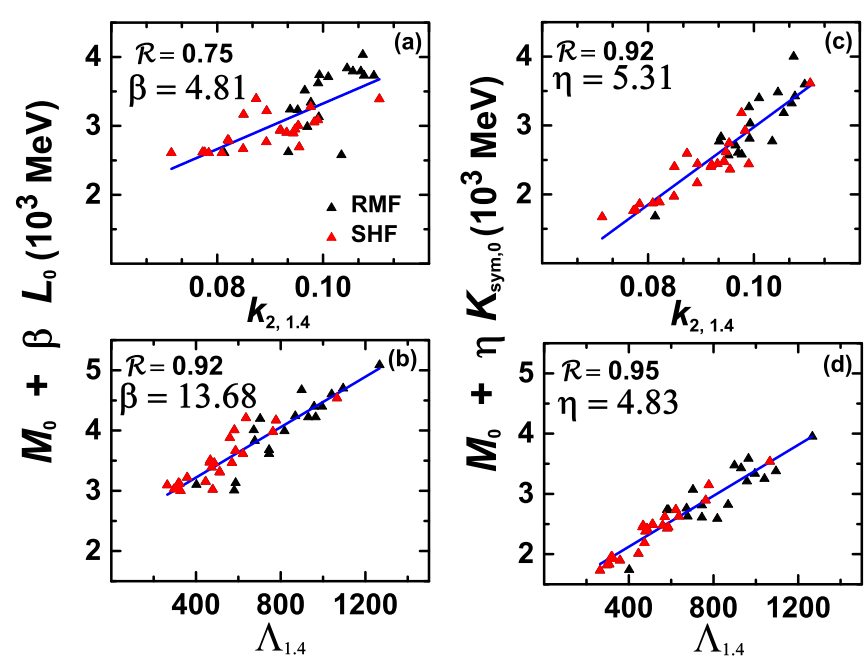

FIG. 5. (a), (b) $M_{0}+\beta L_{0}$ and (c), (d) $M_{0}+\eta K_{\text {sym }, 0}$ versus tidal Love number $k_{2,1.4}$ (top panels) and dimensionless tidal deformability $\Lambda_{1.4}$ (bottom panels) for a $1.4 M_{\odot} \mathrm{NS}$, using a set of RMF and SHF models.

Sec. III A. To check the model dependence of the correlations, we determined the correlation coefficients for the sets of RMF and SHF models separately. The results are given in Table III and indicate that the model dependence is only marginal.

The result for the correlations among $k_{2}, \Lambda$, and various nuclear matter properties as depicted in Fig. 5 may be understood as follows: In Ref. [62], it was shown that the NS radius $R$ is strongly correlated with a linear combination of $M_{0}$ and $L_{0}$ over a wide range of NS masses. This was attributed to the dependence of the pressure on $M_{0}$ and $L_{0}$ and to the empirical relationship of the star radius with the pressure at several reference densities, e.g., $R p(\rho)^{-1 / 4}=$ const. for $\rho \approx 1.5 \rho_{0}$ and NS masses, $1 M_{\odot}-1.4 M_{\odot}$, irrespective of the model [67].

The solid lines in Fig. 5 are obtained using linear regression. These linear regressions yield

$$
\begin{aligned}
\frac{M_{0}}{\mathrm{MeV}}+13.68 \frac{L_{0}}{\mathrm{MeV}}= & (2.09 \pm 0.14) \Lambda_{1.4} \\
& +(2383.12 \pm 96.42), \\
\frac{M_{0}}{\mathrm{MeV}}+4.83 \frac{K_{\mathrm{sym}, 0}}{\mathrm{MeV}}= & (2.11 \pm 0.11) \Lambda_{1.4} \\
& +(1278.13 \pm 77.76) .
\end{aligned}
$$

TABLE III. Values for the correlations coefficients for $\Lambda_{1.4}$ and $k_{2,1.4}$ with $M_{0}+\beta L_{0}$ and $M_{0}+\eta K_{\mathrm{sym}, 0}$ obtained separately for the RMF and SHF models. The values of the correlation coefficients corresponding to all the models (ALL) are also listed.

\begin{tabular}{llllllll}
\hline \hline & \multicolumn{3}{c}{$M_{0}+\beta L_{0}$} & & \multicolumn{3}{c}{$M_{0}+\eta K_{\text {sym }, 0}$} \\
\cline { 2 - 3 } \cline { 6 - 8 } & RMF & SHF & ALL & & RMF & SHF & ALL \\
\hline$\Lambda_{1.4}$ & 0.92 & 0.90 & 0.92 & & 0.88 & 0.97 & 0.95 \\
$k_{2,1.4}$ & 0.72 & 0.68 & 0.75 & & 0.89 & 0.91 & 0.92 \\
\hline \hline
\end{tabular}

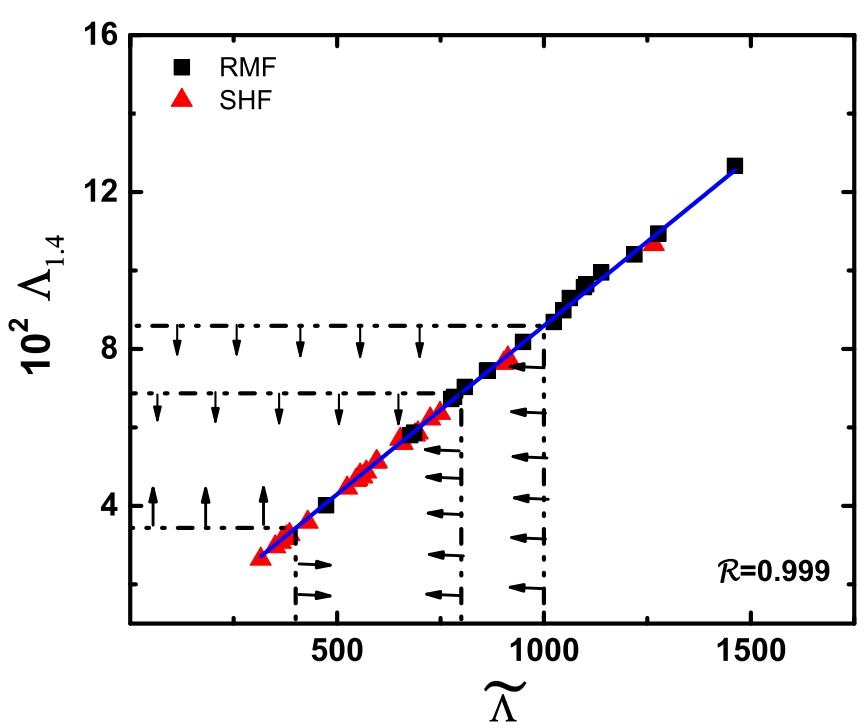

FIG. 6. Tidal deformability $\Lambda_{1.4}$ verses weighted average $\tilde{\Lambda}$ as defined in Eq. (7) for all RMF and SHF models. The solid line represents the best fit. The arrows pointing right and up indicate the lower bounds on $\tilde{\Lambda}$ and $\Lambda_{1.4}$, respectively. The upper bounds on $\tilde{\Lambda}$ and $\Lambda_{1.4}$ are denoted by left and down arrows, respectively.

We need to know the value of $\Lambda_{1.4}$ in order to exploit the correlations, as presented in Fig. 5, to estimate the values of nuclear matter properties at the saturation density.

The GW170817 event provides the upper bound on $\tilde{\Lambda}$ as defined by Eq. (7). For the low-spin prior we have to consider masses such that $q=m_{2} / m_{1}>0.7$. We have calculated the $\tilde{\Lambda}$ using neutron star masses $m=1.4 M_{\odot}, 1.17 M_{\odot}, 1.6 M_{\odot}$, which correspond to the canonical mass and the lower and upper mass limits covered by the analysis of the low-spin prior. The neutron star binary companion mass is determined from the chirp mass $\mathcal{M}=1.188 M_{\odot}: m=1.17 M_{\odot}, 1.6 M_{\odot}$ are, respectively, $m_{2}$ and $m_{1}$ corresponding to $q=0.7$; for the canonical mass we get $q=0.95$ with $m_{1}=1.40 M_{\odot}$ and $m_{2}=1.33 M_{\odot}$. Figure 6 shows the variation of $\Lambda_{1.4}$ as a function of $\tilde{\Lambda}$ for all RMF and SHF models. The correlation between these two quantities is very strong, which enables us to express $\Lambda_{1.4}$ in terms of $\tilde{\Lambda}$ because $\Lambda_{1.4}=0.859 \tilde{\Lambda}$. Similar studies were performed for the NS with mass $m=1.17 M_{\odot}$ and $1.6 M_{\odot}$ and we obtained $\Lambda_{1.17}=2.452 \tilde{\Lambda}$ and $\Lambda_{1.6}=$ $0.379 \tilde{\Lambda}$ with an equally strong correlation. These relations should be compared with the prediction from the expression proposed in Ref. [20]:

$$
\Lambda_{1}=\frac{13}{16} \tilde{\Lambda} \frac{q^{2}(1+q)^{4}}{12 q^{2}-11 q+12},
$$

obtained by replacing

$$
\Lambda_{2}=q^{-6} \Lambda_{1}
$$

in Eq. (7) for $\tilde{\Lambda}$. Equation (15) was obtained by assuming that the radii of the stars with masses $1.17 M_{\odot}<m<1.6 M_{\odot}$ are the same. Using expression (14), we get relations between $\Lambda_{i}$ and $\tilde{\Lambda}$ for $m_{i}=1.17 M_{\odot}, 1.4 M_{\odot}, 1.6 M_{\odot}$ that coincide with ours within the first two digits. We have checked that, in most cases, for our set of models the difference between 


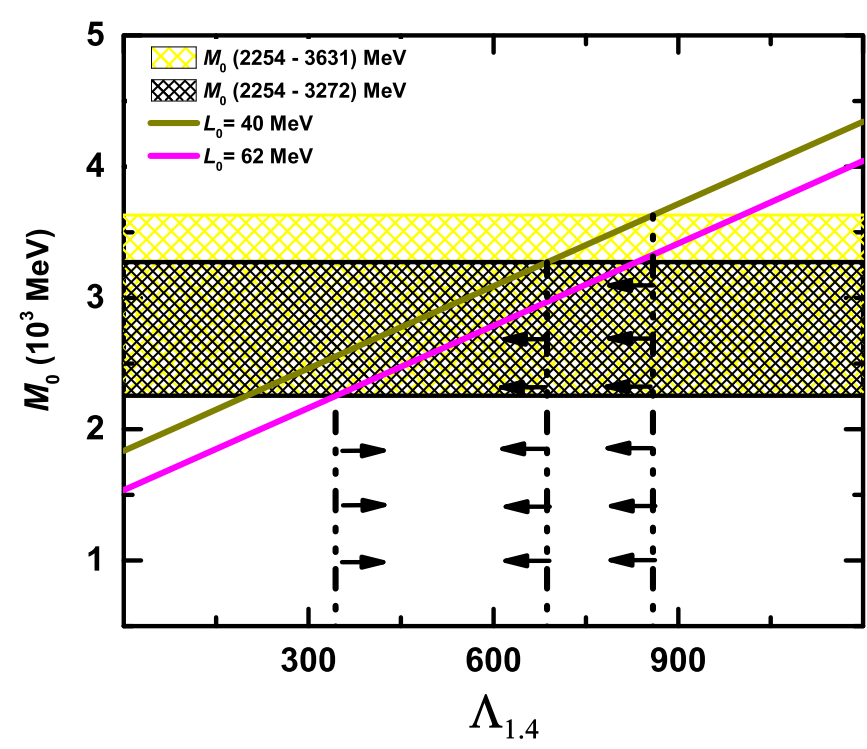

FIG. 7. Plots of incompressibility slope parameter $M_{0}$ versus tidal deformability $\Lambda_{1.4}$ at fixed values of symmetry energy slope parameter $L_{0}$ (solid lines) obtained using Eq. (12). The choices for the values of $L_{0}$ are discussed in the text. The dot-dot-dashed lines represent the bounds obtained in Fig. 6.

the radii of stars with a mass in that interval is not larger than $\approx 0.2 \mathrm{~km}$.

In the following, we want to constraint $M_{0}$ and $K_{\text {sym }, 0}$. We consider the limits imposed on $\Lambda_{1.4}$. This choice is justified because, according to the analysis done in Refs. [20,68], the limits obtained for $\tilde{\Lambda}$ are $q$ dependent and, in particular, in Ref. [20] if the double neutron star or galactic neutron star distributions are considered, the maximum $\tilde{\Lambda}$ value is obtained, respectively, for $q>0.9(q>0.8)$. For the lower limit, the results of Refs. [21,22] were determined for $q>$ 0.85 . A lower bound of $\Lambda_{1.4}>344$ is set by the UV, optical and infrared counterpart of GW170817 that imposes $\tilde{\Lambda}>400$ [21,22]. Similarly, the gravitational-wave observations set an upper bound $\Lambda_{1.4}<687$ or $\Lambda_{1.4}<859$, respectively, from the bounds $\tilde{\Lambda}<800$ [17] and $\tilde{\Lambda}<1000$ [20]. In what follows, we use these bounds on $\Lambda_{1.4}$ together with Eqs. (12) and (13) to constrain the nuclear matter properties.

In Fig. 7, the slope of the incompressibility coefficient at the saturation density $M_{0}$ is plotted as a function of $\Lambda_{1.4}$ for fixed values of $L_{0}$ using Eq. (12). The limiting values of $L_{0}$ employed in the plot correspond to $L_{0}=51 \pm 11 \mathrm{MeV}$ [69]. This limit on $L_{0}$ in conjunction with the bounds on $\Lambda_{1.4}$, as discussed above, constrain $M_{0}$ as listed in Table IV. As referred to before, the lower bound on $\tilde{\Lambda}$ set by Ref. [21] has several associated uncertainties and, therefore, the lower bounds obtained for $M_{0}$ and $K_{\mathrm{sym}, 0}$ suffer from these uncertainties. Notice, however, that independently of the lower value of $\tilde{\Lambda}$ we always have $M_{0}>1500$ (1800) MeV according to the constraints imposed in $L_{0}$ in Ref. [69] ([70]). In the same table we also present the values of $M_{0}$ obtained for $L_{0}=58.7 \pm 28.1 \mathrm{MeV}$ [70]. These values of $L_{0}$ take into account terrestrial, theoretical, and observational constraints. Our values of $M_{0}$ have a reasonable overlap with the values
TABLE IV. The empirical values of $M_{0}$ and $K_{\mathrm{sym}, 0}$ derived for different limits on $\Lambda_{1.4}$ and $L_{0}$. The bounds on $\Lambda_{1.4}>344$ and $<687$ (859) obtained from Fig. 6 are considered. The ranges of $L_{0}=$ $40-62 \mathrm{MeV}$ and $L_{0}=30-86 \mathrm{MeV}$ are taken from Refs. [69,70].

\begin{tabular}{lccc}
\hline \hline $\begin{array}{l}L_{0} \\
(\mathrm{MeV})\end{array}$ & $\Lambda_{1.4}$ & $\begin{array}{c}M_{0} \\
(\mathrm{MeV})\end{array}$ & $\begin{array}{c}K_{\text {sym }, 0} \\
(\mathrm{MeV})\end{array}$ \\
\hline $40-62$ & $344-687$ & $2254-3272$ & $-113--52$ \\
& $344-859$ & $2254-3631$ & $-112--52$ \\
$30-86$ & $344-687$ & $1926-3409$ & $-141-16$ \\
& $344-859$ & $1926-3768$ & $-140-16$ \\
\hline \hline
\end{tabular}

$M_{0}=(1800-2400) \mathrm{MeV}$ obtained empirically in Ref. [71]. The value of $M_{0}$ in Ref. [71] was determined by using a Skyrme-like energy density functional by imposing the constraint on the incompressibility slope parameter at the crossing density $\left(\approx 0.1 \mathrm{fm}^{-3}\right)$ determined from energies of the isoscalar giant monopole resonance in the ${ }^{132} \mathrm{Sn}$ and ${ }^{208} \mathrm{~Pb}$ nuclei $[72,73]$.

The above analysis is dependent of the star mass used to calculate the tidal deformability. However, it is important to note that the contribution of $M_{0}$ to the linear combination $M_{0}+\beta L_{0}$ is maximum for the larger star masses, so large star masses that satisfy the $q$ constraints should be chosen. Taking $\Lambda_{1.6}(q=0.7)$ to constraint $M_{0}$, the upper limits would have been $\approx 5 \%-10 \%$ lower.

We next consider the range of acceptable values for $M_{0}$ just determined, together with the bounds on $\Lambda_{1.4}$ and Eq. (13), to set also constraints on $K_{\mathrm{sym}, 0}$. The results are presented in Table IV: the ranges $-113<K_{\mathrm{sym}, 0}<-52 \mathrm{MeV}$ are obtained for the constraints on the symmetry energy slope from Ref. [69] and $-141<K_{\text {sym }, 0}<16 \mathrm{MeV}$ imposing the constraints from Ref. [70]. The symmetry energy curvature is a quantity that is still not constrained experimentally. In Ref. [74], the authors have obtained from the universality of the correlation structure between the different symmetry energy elements and from some well-known nuclear matter properties the range $K_{\mathrm{sym}, 0}=-111.8 \pm 71.3 \mathrm{MeV}$. Our bounds discussed above are in a quite good agreement with these values.

Figure 8 displays the tidal Love number $k_{2,1.4}$ (top panel) and the dimensionless tidal deformability $\Lambda_{1.4}$ (bottom panel) as a function of NS radius $R_{1.4}$. It is evident from Eq. (2) that the tidal deformability depends mainly on the NS radius and the Love number $k_{2} . \Lambda_{1.4}$ is expected to be strongly correlated with $R_{1.4}$ provided either $k_{2}$ is model independent or it is correlated with $R_{1.4}$. We observe from Fig. 2 that the value of $k_{2}$ is sensitive to the model used, which might influence the correlation between $\Lambda_{1.4}$ and $R_{1.4}$. However, $k_{2,1.4}$ is moderately correlated with $R_{1.4}$ (top panel) which ensures the persistence of the strong correlation $(\mathcal{R}=0.98)$ between $\Lambda_{1.4}$ and $R_{1.4}$ (bottom panel). The solid line in the bottom panel represent the fitted curve with equation $\Lambda_{1.4}=$ $9.11 \times 10^{-5}\left(\frac{R_{1.4}}{\mathrm{~km}}\right)^{6.13}$. This equation can be rewritten in a form similar to the relation obtained in Ref. [20], which expresses the tidal deformability in terms of the compactness parameter of the star, $\beta=G m /\left(R c^{2}\right)$,

$$
\Lambda=a \beta^{-6},
$$




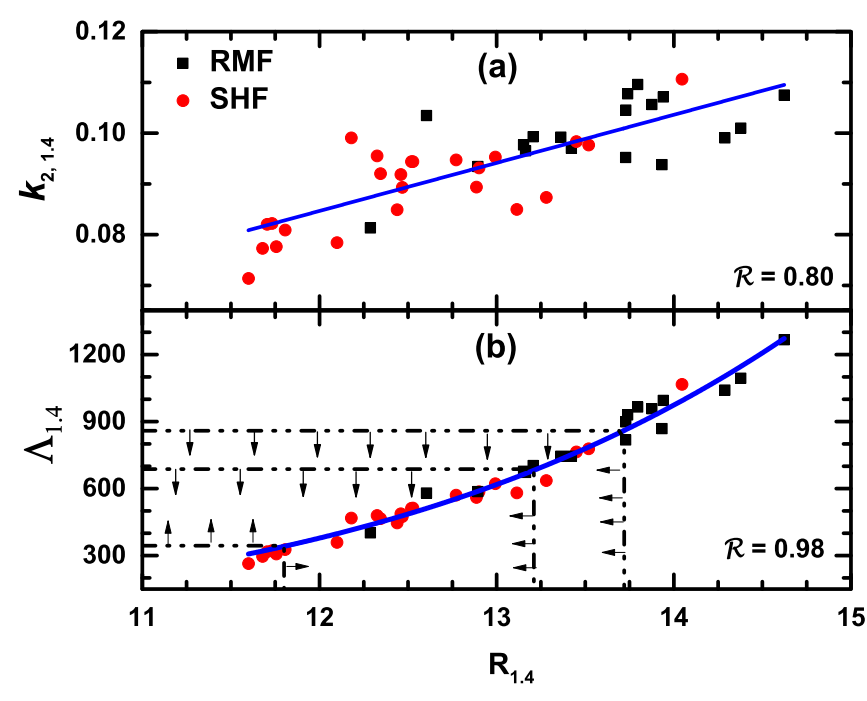

FIG. 8. (a) Variation of tidal Love number $k_{2,1.4}$ and (b) dimensionless tidal deformability $\Lambda_{1.4}$ with radius $R_{1.4}$ obtained from RMF (black squares) and SHF (red circles) models. The solid lines in the top and bottom panels are the best-fit linear and curve lines, respectively. The horizontal dot-dot-dashed lines represent the bounds obtained in Fig. 6.

having the exponent 6.13 instead of 6 . We verified that the exponent is mass dependent although close to 6: taking $m=$ $1.17 M_{\odot}$ and $m=1.60 M_{\odot}$ the exponent is, respectively, 5.84 and 6.58. In our analysis we use a set of models different from that used in Ref. [20] and, besides, we have only considered unified inner crust-core EoS, while in Ref. [20] all the EoSs have a common crust EoS. These two aspects could explain some of the differences. Using the derived bounds on $\Lambda_{1.4}$, the value of $R_{1.4}$ is found to be in the range 11.82-13.22 (11.82-13.72) km for $\Lambda_{1.4}$ in the range of 344-687 (344859). These ranges for $R_{1.4}$ lie almost within the bounds of $8-14 \mathrm{~km}$ and $10.5-13.3 \mathrm{~km}$ as estimated from GW170817 in Refs. [20,68]. Furthermore, our predictions are in harmony with $R_{1.4}=11.5-13.6 \mathrm{~km}$ [75] as constrained by the slope of the symmetry energy which was extracted by using the terrestrial laboratory data on the isospin diffusion in heavy-ion reactions at intermediate energies.

\section{CONCLUSIONS}

The recent observation of GW170817 has provided an upper bound on tidal deformability parameter. Complementing the gravitational wave observation with the detection of the UV, optical and infrared counterpart of GW170817, a lower bound on the tidal deformability parameter is suggested [21]. We used a diverse set of relativistic and nonrelativistic mean-field models to look for correlations of $\Lambda$ with several nuclear matter parameters characterizing the EoS, such as the nuclear matter incompressibility and symmetry energy coefficients, and their density derivatives. All the models selected are consistent with the bulk properties of finite nuclei as well as with the observation of NSs with mass of $\approx 2 M_{\odot}$. Nevertheless, across these models, the values of $\Lambda$ and of the various nuclear matter parameters associated with different EoSs vary over a wide range.

The tidal deformability is found to be weakly or only moderately correlated with the individual nuclear matter parameters of the EoS. The stronger correlation of $\Lambda$ is found only for specific choices of the linear combinations of the isoscalar and isovector EoS parameters. The parameter $\Lambda$ is strongly correlated with the linear combination of the slopes of incompressibility and symmetry energy coefficients, i.e., $M_{0}+\beta L_{0}$. Furthermore, the parameter $\Lambda$ and the Love number $k_{2}$ both are strongly correlated with the linear combination of $M_{0}+\eta K_{\mathrm{sym}, 0}$.

We show that the bound on weighted average of tidal deformability for a system of binary neutron stars, obtained from complementary analysis $[17,20,21]$ of GW170817, yields the tidal deformability for NSs with mass $1.4 M_{\odot}$ in the range of $344<\Lambda_{1.4}<859$. With the aid of the correlations of $\Lambda_{1.4}$ with linear combinations of nuclear matter parameters as considered together with the bounds on $\Lambda_{1.4}$ and the empirical ranges of $L_{0}$ obtained in Refs. [69,70], we have constrained the values of $M_{0}$ and $K_{\mathrm{sym}, 0}$ to lie in the intervals $2254<$ $M_{0}<3631 \mathrm{MeV}$ and $-112<K_{\text {sym }, 0}<-52 \mathrm{MeV}$ or $1926<$ $M_{0}<3768 \mathrm{MeV}$ and $-140<K_{\text {sym }, 0}<16 \mathrm{MeV}$, depending on the constraints set on $L_{0}$. The strong correlation of tidal deformability with the NS radius for a $1.4 M_{\odot}$ NS yields $R_{1.4}$ in the range $11.82-13.72 \mathrm{~km}$. The precise measurement of tidal deformability will provide an alternative and accurate estimate for $M_{0}, K_{\mathrm{sym}, 0}$, and $R_{1.4}$.

\section{ACKNOWLEDGMENTS}

T.M. is grateful to the Theory Group, Saha Institute of Nuclear Physics for the hospitality accorded to him during the phase of this work. The work of M.F. has

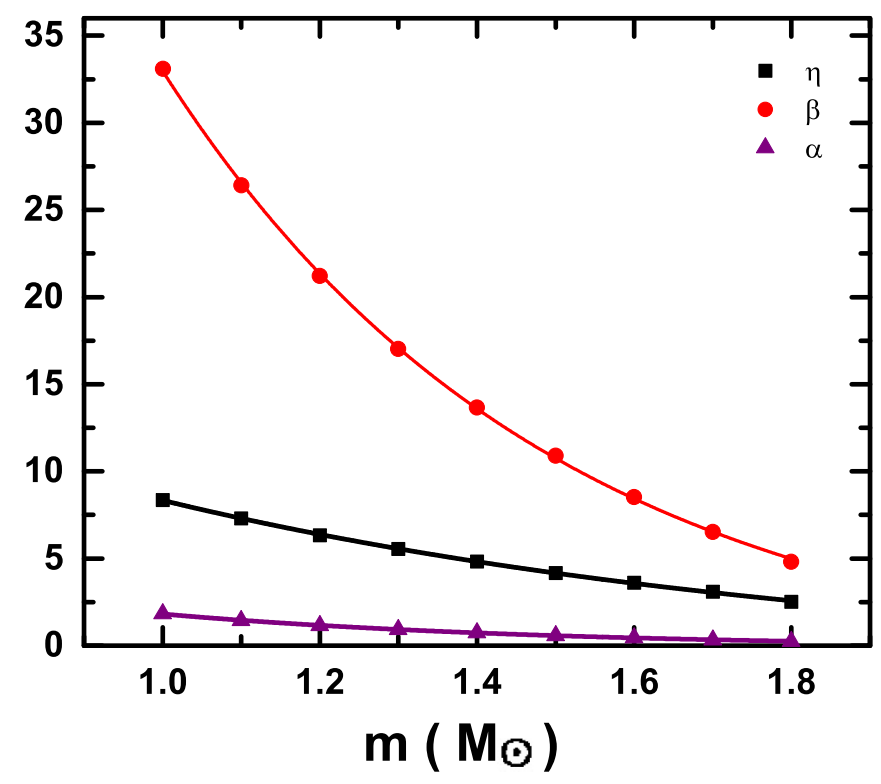

FIG. 9. The values of $\alpha, \beta$, and $\eta$ obtained by optimizing the the correlations of $\Lambda$ with the linear combinations $K_{0}+\alpha L_{0}, M_{0}+$ $\beta L_{0}$, and $M_{0}+\eta K_{\mathrm{sym}, 0}$ are plotted as a function of NS mass. 
been partially supported by the NCN (Poland) Grant No. 2014/13/B/ST9/02621 and by a STSM grant from the COST action CA16214 "PHAROS." C.P. acknowledges financial support by Fundação para a Ciência e Tecnologia (FCT) Portugal under project No. UID/FIS/04564/2016, project POCI01-0145-FEDER-029912 with financial support from POCI, in its FEDER component, and by the FCT/MCTES budget through national funds (OE), and the COST action CA16214 "PHAROS." T.K.J. \& T.M. thank DAE-BRNS for its support (2013/37P/5/BRNS). B.K. would like to thank P. Landry and Kenta Hotokezaka for useful discussions. N.A. is partially supported by Indo-French CEFIPRA project.

\section{APPENDIX: MASS DEPENDENCE OF $\alpha, \beta$, AND $\eta$}

The coefficients $\alpha, \beta$, and $\eta$ are obtained in such a way that they optimize the correlations of $\Lambda$, for a given NS mass, with the linear combinations $K_{0}+\alpha L_{0}, M_{0}+\beta L_{0}$, and $M_{0}+\eta K_{\text {sym }, 0}$. The value of these coefficients are given in Table II for a few selected NS masses. In Fig. 9, we plot the mass dependence of $\alpha, \beta$, and $\eta$. These coefficients can be easily fit to the exponential decay like function which can be expressed as $\alpha=-0.13+14.87 \exp (-m / 0.49)$, $\beta=-1.90+265.02 \exp (-m / 0.49), \quad$ and $\quad \eta=-1.4+$ $29.81 \exp (-m / 0.89)$, where the NS mass $m$ is in units of solar mass.
[1] P. Haensel, A. Y. Potekhin, and D. G. Yakovlev, Neutron Stars 1: Equation of State and Structure (Springer, New York, 2007), Vol. 326.

[2] J. Antoniadis et al., Science 340, 1233232 (2013).

[3] Z. Arzoumanian, A. Brazier, S. Burke-Spolaor, S. Chamberlin et al., Astrophys. J., Suppl. Ser. 235, 37 (2018).

[4] J. H. Taylor and J. M. Weisberg, Astrophys. J. 253, 908 (1982).

[5] R. W. P. Drever, Gravitational Radiation (North-Holland, Amsterdam, 1983), p. 321.

[6] K. Thorne, Three Hundred Years of Gravitation, edited by S. W. Hawking and W. Israel (Cambridge University Press, Cambridge, U.K., 1987), pp. 330-458.

[7] A. Brillet et al. (Virgo Collaboration), Technical Report No. VIR-0517A-15 (1989), https://tds.virgo-gw.eu/?content=3 $\& \mathrm{r}=12122$.

[8] J. Hough et al., MPQ Technical Report No. 147 [GWD/137/JH(89)] (1989).

[9] C. Cutler, T. A. Apostolatos, L. Bildsten, L. S. Finn, E. E. Flanagan, D. Kennefick, D. M. Markovic, A. Ori, E. Poisson, G. J. Sussman, and K. S. Thorne, Phys. Rev. Lett. 70, 2984 (1993).

[10] J. Faber, Classical Quantum Gravity 26, 114004 (2009).

[11] M. D. Duez, Classical Quantum Gravity 27, 114002 (2010).

[12] E. E. Flanagan and T. Hinderer, Phys. Rev. D 77, 021502 (2008).

[13] T. Hinderer, Astrophys. J. 677, 1216 (2008).

[14] T. Damour and A. Nagar, Phys. Rev. D 80, 084035 (2009).

[15] T. Binnington and E. Poisson, Phys. Rev. D 80, 084018 (2009).

[16] T. Hinderer, B. D. Lackey, R. N. Lang, and J. S. Read, Phys. Rev. D 81, 123016 (2010).

[17] B. P. Abbott et al. (LIGO Scientific Collaboration and Virgo Collaboration), Phys. Rev. Lett. 119, 161101 (2017).

[18] P. S. Cowperthwaite et al., Astrophys. J. Lett. 848, L17 (2017).

[19] V. A. Villar et al., Astrophys. J. Lett. 851, L21 (2017).

[20] S. De, D. Finstad, J. M. Lattimer, D. A. Brown, E. Berger, and C. M. Biwer, Phys. Rev. Lett. 121, 091102 (2018).

[21] D. Radice, A. Perego, F. Zappa, and S. Bernuzzi, Astrophys. J. Lett. 852, L29 (2018).

[22] A. Perego, D. Radice, and S. Bernuzzi, Astrophys. J. Lett. 850, L37 (2017).

[23] F. J. Fattoyev, J. Carvajal, W. G. Newton, and B.-A. Li, Phys. Rev. C 87, 015806 (2013).

[24] F. J. Fattoyev, J. Piekarewicz, and C. J. Horowitz, Phys. Rev. Lett. 120, 172702 (2018).
[25] N. Alam, A. Sulaksono, and B. K. Agrawal, Phys. Rev. C 92, 015804 (2015).

[26] M. Fortin, C. Providência, A. R. Raduta, F. Gulminelli, J. L. Zdunik, P. Haensel, and M. Bejger, Phys. Rev. C 94, 035804 (2016).

[27] N.-B. Zhang, B.-A. Li, and J. Xu, Astrophys. J. Lett. 859, 90 (2018).

[28] T. Damour, A. Nagar, and L. Villain, Phys. Rev. D 85, 123007 (2012)

[29] S. Postnikov, M. Prakash, and J. M. Lattimer, Phys. Rev. D 82, 024016 (2010).

[30] S. Weinberg, Gravitation and Cosmology (Wiley, New York, 1972).

[31] M. Favata, Phys. Rev. Lett. 112, 101101 (2014).

[32] I. Vidaña, C. Providência, A. Polls, and A. Rios, Phys. Rev. C 80, 045806 (2009).

[33] S. K. Dhiman, R. Kumar, and B. K. Agrawal, Phys. Rev. C 76, 045801 (2007).

[34] B. K. Agrawal, Phys. Rev. C 81, 034323 (2010).

[35] W.-C. Chen and J. Piekarewicz, Phys. Rev. C 90, 044305 (2014)

[36] N. K. Glendenning and S. A. Moszkowski, Phys. Rev. Lett. 67, 2414 (1991).

[37] G. A. Lalazissis, J. König, and P. Ring, Phys. Rev. C 55, 540 (1997).

[38] H. Pais and C. Providência, Phys. Rev. C 94, 015808 (2016).

[39] C. J. Horowitz and J. Piekarewicz, Phys. Rev. Lett. 86, 5647 (2001).

[40] J. Carriere, C. Horowitz, and J. Piekarewicz, Astrophys. J. 593, 463 (2003).

[41] Y. Sugahara and H. Toki, Nucl. Phys. A 579, 557 (1994).

[42] C. Providência and A. Rabhi, Phys. Rev. C 87, 055801 (2013).

[43] S. Typel, G. Ropke, T. Klahn, D. Blaschke, and H. H. Wolter, Phys. Rev. C 81, 015803 (2010).

[44] T. Gaitanos, M. Di Toro, S. Typel, V. Baran, C. Fuchs, V. Greco, and H. H. Wolter, Nucl. Phys. A A732, 24 (2004).

[45] C. Ducoin, J. Margueron, C. Providência, and I. Vidaña, Phys. Rev. C 83, 045810 (2011).

[46] T. Nikšić, D. Vretenar, P. Finelli, and P. Ring, Phys. Rev. C 66, 024306 (2002).

[47] G. A. Lalazissis, T. Nikšić, D. Vretenar, and P. Ring, Phys. Rev. C 71, 024312 (2005).

[48] S. Typel and H. H. Wolter, Nucl. Phys. A 656, 331 (1999).

[49] H. Kohler, Nucl. Phys. A 258, 301 (1976). 
[50] P. G. Reinhard and H. Flocard, Nucl. Phys. A 584, 467 (1995).

[51] W. Nazarewicz, J. Dobaczewski, T. R. Werner, J. A. Maruhn, P.-G. Reinhard, K. Rutz, C. R. Chinn, A. S. Umar, and M. R. Strayer, Phys. Rev. C 53, 740 (1996).

[52] E. Chabanat, Ph.D. thesis, University Claude Bernard Lyon-1, Lyon, France, 1995 (unpublished).

[53] E. Chabanat, P. Bonche, P. Haensel, J. Meyer, and R. Schaeffer, Nucl. Phys. A 627, 710 (1997).

[54] E. Chabanat, P. Bonche, P. Haensel, J. Meyer, and R. Schaeffer, Nucl. Phys. A 635, 231 (1998).

[55] L. Bennour, P. H. Heenen, P. Bonche, J. Dobaczewski, and H. Flocard, Phys. Rev. C 40, 2834 (1989).

[56] P. G. Reinhard, Nucl. Phys. A 649, 305c (1999).

[57] B. K. Agrawal, S. Shlomo, and V. K. Au, Phys. Rev. C 72, 014310 (2005).

[58] B. K. Agrawal, S. Shlomo, and A. I. Sanzhur, Phys. Rev. C 67, 034314 (2003).

[59] J. Friedrich and P.-G. Reinhard, Phys. Rev. C 33, 335 (1986).

[60] S. Goriely, N. Chamel, and J. M. Pearson, Phys. Rev. C 82, 035804 (2010).

[61] S. Goriely, N. Chamel, and J. M. Pearson, Phys. Rev. C 88, 024308 (2013).
[62] N. Alam, B. K. Agrawal, M. Fortin, H. Pais, C. Providência, A. R. Raduta, and A. Sulaksono, Phys. Rev. C 94, 052801 (2016).

[63] M. Fortin, S. S. Avancini, C. Providência, and I. Vidaña, Phys. Rev. C 95, 065803 (2017).

[64] G. Baym, C. Pethick, and P. Sutherland, Astrophys. J. 170, 299 (1971).

[65] S. Brandt, Statistical and Computational Methods in Data Analysis, 3rd English ed. (Springer, New York, 1997).

[66] C. J. Horowitz and J. Piekarewicz, Phys. Rev. C 64, 062802(R) (2001).

[67] J. M. Lattimer and M. Prakash, Astrophys. J. 550, 426 (2001).

[68] B. P. Abbott et al. (Virgo and LIGO Scientific Collaboration), arXiv:1805.11581.

[69] J. M. Lattimer and Y. Lim, Astrophys. J. 771, 51 (2013).

[70] M. Oertel, M. Hempel, T. Klähn, and S. Typel, Rev. Mod. Phys. 89, 015007 (2017).

[71] J. N. De, S. K. Samaddar, and B. K. Agrawal, Phys. Rev. C 92, 014304 (2015).

[72] E. Khan, J. Margueron, and I. Vidaña, Phys. Rev. Lett. 109, 092501 (2012).

[73] E. Khan and J. Margueron, Phys. Rev. C 88, 034319 (2013).

[74] C. Mondal, B. K. Agrawal, J. N. De, S. K. Samaddar, M. Centelles, and X. Viñas, Phys. Rev. C 96, 021302 (2017).

[75] B.-A. Li and A. W. Steiner, Phys. Lett. B 642, 436 (2006). 